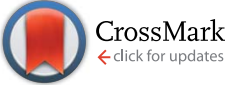

Cite this: RSC Adv., 2015, 5, 54510

\title{
Influence of PCL molecular weight on mesenchymal stromal cell differentiation
}

\author{
W. J. Hendrikson, ${ }^{a}$ J. Rouwkema, ${ }^{b}$ C. A. van Blitterswijk ${ }^{\text {ac }}$ and L. Moroni ${ }^{\star a c}$
}

Regenerating or replacing bone, chondral and osteochondral defects, is an active field in tissue engineering. A general strategy is to use a temporary scaffold in which cells are seeded onto the scaffold prior to implantation or attracted into the scaffold from surrounding tissues in the implantation site to form the desired tissue. Several biomaterials have been used for the fabrication of scaffolds, including polycaprolactone (PCL) which is often used for musculoskeletal tissue engineering. The effect of the $\mathrm{PCL}$ scaffold architecture on the cell behavior has been investigated. However, the mechanical properties of the bulk material were not taken into account in these studies. PCL is available in a range of molecular weights, resulting in a range of bulk mechanical properties. Since bulk material stiffness is able to direct cell differentiation, it is likely that the molecular weight of PCL may influence cell behavior. Here, we investigated the bulk material properties of both low and high molecular weight PCL scaffolds fabricated through additive manufacturing. The low molecular weight PCL showed a lower bulk material stiffness. During in vitro cell culture, this resulted in a stronger tendency for hypertrophic chondrogenic differentiation compared to the high molecular weight PCL. This study shows that apart from the polymer chemistry and scaffold architecture, the bulk mechanical properties of the polymer used is an important parameter in scaffold fabrication. This is an important finding for the optimization of osteochondral tissue engineering.

Received 1st May 2015

Accepted 16th June 2015

DOI: $10.1039 / \mathrm{c} 5 \mathrm{ra08048g}$

www.rsc.org/advances be biocompatible and biodegradable, while the mechanical properties of the scaffold should ideally mimic the mechanical properties of the native tissue and simultaneously shield the forming tissue from excessive mechanical loading. Furthermore, the mechanical properties of the scaffold could be used to elicit a specific cellular response when biophysically loaded. The properties of the scaffold should be tailored to match the formation of the new tissue in the scaffold. ${ }^{8}$ A ceramic, a natural-, or a synthetic polymer is often used to fabricate scaffolds for tissue engineering purposes. Advantages of ceramic and natural polymers are that their intrinsic properties can elicit cellular responses on their own. However, processing of these materials to fabricate scaffolds with controlled pore shapes and porosity is often more difficult compared to synthetic polymers. ${ }^{5,9,10}$ Although synthetic polymers in general don't possess intrinsic properties to elicit cellular response, the ability to tailor the material to display desirable properties makes them very interesting. Due to this, synthetic polymers like polycaprolactone (PCL), poly lactic acid (PLA) and poly glycolic acid (PGA) are often used as the biomaterial for scaffolds. ${ }^{711-14}$ All of these biodegradable synthetic polymers have found their way into clinical applications and are well described in literature. Especially PCL has a long track record in biomedical applications, as also well described in a review by Woodruff and Hutmacher. ${ }^{15}$ In this study, we use PCL scaffolds obtained through additive manufacturing (AM). This
${ }^{a}$ Department of Tissue Regeneration, MIRA Institute for Biomedical Technology and Technical Medicine, University of Twente, Enschede, 7500 AE, The Netherlands

${ }^{b}$ Department of Biomechanical Engineering, MIRA Institute for Biomedical Technology and Technical Medicine, University of Twente, Enschede, 7500 AE, The Netherlands ${ }^{c}$ Complex Tissue Regeneration Department, MERLN Institute for Technology Inspired Regenerative Medicine, University of Maastricht, Maastricht, 6229 ER, The Netherlands. E-mail: l.moroni@maastrichtuniversity.nl 
technology allows for the control of the scaffold geometry, and thereby enables the fabrication of patient specific scaffolds. Apart from that, the scaffold internal architecture can be controlled and reproduced. As such, possible effect of the fabrication technique used on cell behaviour can be eliminated. ${ }^{16,17}$

Because PCL is such a common material, PCL of different molecular weights can be obtained. The surface chemistry and topography is expected to be the same when obtaining PCL scaffolds through the same production technique. However, the bulk stiffness of the different molecular weights will be different. From literature, it is known that the bulk material stiffness can influence cell behaviour. ${ }^{18-20}$ In these studies, hydrogels with a stiffness in the range of 1-100 kPa were generally used as biomaterials. Cells were either cultured on 2D hydrogels, ${ }^{21}$ coated with collagen type $\mathrm{I}^{19,20}$ or encapsulated in the hydrogel. ${ }^{22,23}$ To the authors' knowledge, the effect of mechanical properties of stiffer polymers, such as PCL, on cells has not been investigated. Additionally, the effect of bulk material stiffness on cells in an open porous 3D scaffold is not known. We hypothesize that by using PCL with different molecular weights, a difference in behaviour of mesenchymal stromal cells can be observed.

\section{Materials and methods}

\section{Scaffold fabrication}

Scaffolds were fabricated by a BioScaffolder (SYSeng, Germany), as previously described. ${ }^{24}$ Briefly, granules of either low PCL $\left(M_{\mathrm{w}}\right.$ 14.000, Sigma) or high PCL ( $M_{\mathrm{w}} 65.000$, Sigma) were inserted in the cartridge of the machine and heated to $60-65^{\circ} \mathrm{C}$ for low PCL and to $125-130{ }^{\circ} \mathrm{C}$ for high PCL. An applied nitrogen pressure of 5 bar and an auger screw system rotating at 200 RPM extruded the molten polymer through a $250 \mu \mathrm{m}$ diameter needle (DL technology) on a stationary platform. Scaffolds were fabricated through a layer-by-layer deposition, in which the angle between layers, fiber spacing and layer thickness can be set while the fiber diameter depends on the needle size chosen. Scaffold blocks of $30 \times 30 \times 2.1 \mathrm{~mm}$ were created with a fiber diameter of 175-200 $\mu \mathrm{m}$, a layer thickness of $150 \mu \mathrm{m}$, a fiber spacing of $1000 \mu \mathrm{m}$ and $90^{\circ}$ angle between layers. Cylindrical scaffolds of $8 \mathrm{~mm}$ in diameter were obtained by using a biopsy puncher (Miltex). The actual fiber spacing and fiber diameter were measured with Image J. For this, stereomicroscope images were loaded and measurements were performed at random locations (3 samples, 5 measurements per sample).

\section{Mechanical characterization of low and high PCL}

Solid blocks of low and high PCL were obtained through compression molding to determine the stiffness of the bulk material. Polymer granules of either low or high PCL were placed in an open, heated mold cavity, $50 \mathrm{~mm}$ diameter and $2 \mathrm{~mm}$ high. After closing the mold with a plug, pressure was applied and the mold was heated until the polymer reached the molten phase. The mold was pressurized and heated for 5 minutes, after which the samples were cooled to room temperature. Three cylindrical plugs of $6 \mathrm{~mm}$ diameter were punched from the blocks and used for determination of the bulk material stiffness. An unconfined compression test was performed with a Zwick Z050. A compression of 5\% strain per minute to a maximum of $15 \%$ strain was applied. Stresses at $4 \%$ and $8 \%$ strain were used to calculate the stiffness.

\section{Cell culture}

Bone marrow aspirates were obtained from patients after written informed consent. hMSCs were isolated and proliferated as described previously. ${ }^{25}$ Basic medium consisted of $\alpha$-MEM (Gibco) supplemented with 10\% FBS (Lonza), $0.2 \mathrm{mM}$ L-ascorbic acid (Sigma), $2 \mathrm{mM}$ L-glutamine (Gibco), $100 \mathrm{U} \mathrm{mL}^{-1}$ penicillin (Life Technologies) and $100 \mu \mathrm{g} \mathrm{mL}{ }^{-1}$ streptomycin (Life Technologies). Proliferation medium was basic medium supplemented with $1 \mathrm{ng} \mathrm{mL}^{-1}$ bFGF (Instruchemie). Osteogenic differentiation medium consisted of basic medium supplemented with $10 \mathrm{nM}$ dexamethasone (Sigma). Chondrogenic differentiation medium consisted of high glucose D-MEM (Gibco) supplemented with $50 \mathrm{mg} \mathrm{mL}^{-1}$ ITS pre-mix (BD biosciences), 50 $\mathrm{mg} \mathrm{mL} \mathrm{m}^{-1} \mathrm{~L}$-ascorbic acid (Sigma), $100 \mathrm{mg} \mathrm{mL}^{-1}$ sodium pyruvate (Sigma), $100 \mathrm{U} \mathrm{mL}^{-1}$ penicillin (Invitrogen) and $100 \mu \mathrm{g} \mathrm{mL}$

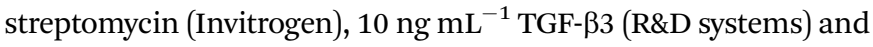
$0.1 \mathrm{nM}$ dexamethasone, which were freshly added every time that medium was changed. Cells were grown up to $80-90 \%$ confluency at $37^{\circ} \mathrm{C}$ in a humidified atmosphere with $5 \% \mathrm{CO}_{2}$. Passage 3 cells were used for all experiments.

\section{Cell culture on scaffolds}

Scaffolds were sterilized by immersion in $70 \%$ ethanol, twice for at least 15 minutes, and were subsequently washed with PBS. Afterwards, the sterile scaffolds were immersed in a $1 \mathrm{mg} \mathrm{mL}^{-1}$ rat tail collagen type I (BD biosciences) solution overnight in a cell culture incubator. Scaffolds were washed with PBS and dried with the aspiration pump shortly before seeding 500.000 hMSCs per scaffold in $65 \mu \mathrm{L}$ proliferation medium. hMSCs for chondrogenic differentiation were pre-incubated with $0.3 \mathrm{mg} \mathrm{mL}^{-1}$ fibronectin (Invitrogen) for 15 minutes before seeding. After seeding, cells were allowed to attach to the scaffold surface for 4 $\mathrm{h}$ before adding proliferation medium to $2 \mathrm{~mL}$ per scaffold. All scaffolds were cultured in proliferation medium for 7 days before changing to either basic, osteogenic or chondrogenic differentiation medium. Scaffolds were removed from cell culture after 7 , 14 and 28 days of culture in differentiation media. At each time point, 3 scaffolds were collected for biochemical analysis, 1 for histology and 1 for microscopy. Every other day the medium was refreshed. The cell seeded scaffolds were placed in an incubator at $37^{\circ} \mathrm{C}$ in a humidified atmosphere with $5 \% \mathrm{CO}_{2}$.

\section{DNA assay}

Cell cultured scaffolds $(N=3)$ were cut in half for either a.u. ALP/ $/ \mu$ D DNA quantification or $\mu g \mathrm{GAG} / \mu \mathrm{g}$ DNA quantification. Each half was cut in as small pieces as possible and subjected to a cycle of freeze-thawing 5 times before analysis. Total DNA content was determined, on either lysate, following the manufacturer's protocol (CyQuant Cell proliferation assay kit). ALP and GAG were corrected by their total DNA content. 


\section{ALP assay}

Cell lysis buffer (0.1 $\mathrm{M} \mathrm{KH}_{2} \mathrm{PO}_{4}, 0.1 \mathrm{M} \mathrm{K}_{2} \mathrm{HPO} 4,0.1 \%$ Triton $\mathrm{X}-100, \mathrm{pH} 7.8$ ) was added to the cut samples and incubated for 1 hour at room temperature before ALP or DNA quantification. ALP activity was quantified from the lysate according to the manufacturer's protocol (CDP-star Chemiluminescent substrate kit, Roche) with a VICTOR plate reader (Perkin-Elmer).

\section{GAG assay}

The other half of the cell cultured scaffold was digested in 1 $\mathrm{mg} \mathrm{mL} \mathrm{m}^{-1}$ proteinase $\mathrm{K}$ (Sigma) in Tris/EDTA ( $\mathrm{pH}$ 7.6) containing $18.5 \mu \mathrm{g} \mathrm{mL}^{-1}$ iodoacetamine (Sigma) and $1 \mu \mathrm{g} \mathrm{mL}^{-1}$ pepstatin A (Sigma) for 16 hours at $56{ }^{\circ} \mathrm{C}$. Sulphated GAG content was spectrophotometrically determined immediately after adding 9-dimethylmethylene blue dye (DMMB, $3.04 \mathrm{~g} \mathrm{~L}^{-1}$ glycine, $2.37 \mathrm{~g} \mathrm{~L}^{-1}$ of $\mathrm{NaCl}, \mathrm{pH}=3$ ) at an absorbance of $525 \mathrm{~nm}$ in a Multiskan Go (Perkin-Elmer). The amount of GAG was determined using a calibration curve of chondroitin sulphate.

\section{Methylene blue and ALP staining}

Cell seeded scaffolds were washed with PBS and fixated by $10 \%$ neutral buffered formalin for 1 hour. Scaffolds were cut in half and one part was used for methylene blue and the other for ALP staining. A drop of 1\% methylene blue solution was applied to the scaffold for one minute. The stained scaffolds were washed with demineralized water until there was no more discolouring of the water. Samples were imaged with a Nikon SMZ-10A stereomicroscope equipped with a Sony 3CCD camera.

ALP staining was conducted with the Leukocyte Alkaline Phosphatase kit (Sigma) following the manufacturers protocol. The remaining halves of the scaffolds were incubated with the fresh prepared alkaline dye for 30 minutes, after which the scaffolds were washed with demineralized water and imaged with a Nikon stereomicroscope.

\section{Scanning electron microscopy (SEM)}

Formalin fixated scaffold samples were dehydrated through an ethanol series. The samples were immersed in HMDS (Sigma) for 15 minutes after total dehydration. Excessive HMDS was removed and the samples were left to dry overnight. The dried samples were mounted on a stage, gold sputtered and imaged with a SEM (XL-30 ESEM-FEG, Phillips).

\section{Statistical analysis}

A two way ANOVA with Bonferroni's post-hoc test was used. $P$ values less than 0.05 were considered to be statistically significant. All values are reported as average mean with standard deviation.

\section{Results}

\section{Mechanical characterization}

Two types of PCL were chosen based on their molecular weight. Compression moulded cylindrical blocks were subjected to an unconfined compression. The determined stiffness was $204.2 \pm$ 8.6 MPa for high PCL and 146.5 \pm 7.6 MPa for low PCL.

\section{Scaffold characterization}

3D PCL scaffolds with pre-defined architectures were obtained through additive manufacturing. Measurements showed an actual fiber spacing of $1068 \pm 23 \mu \mathrm{m}$, a fiber diameter of $213 \pm 26 \mu \mathrm{m}$ and a layer height of $159 \pm 14 \mu \mathrm{m}$ for low PCL scaffolds. For high PCL scaffolds, the fiber spacing was $1059 \pm 22 \mu \mathrm{m}$, the fiber diameter was $187 \pm 18 \mu \mathrm{m}$ and the layer thickness was $151 \pm 7 \mu \mathrm{m}$.

\section{Cell distribution and ECM formation}

Methylene blue images showed good cell attachment for both low and high PCL and for all media (Fig. 1). Cell seeded scaffolds cultured in chondrogenic differentiation media showed rounded cell morphology (Fig. 1M-R). Homogeneous cell distribution was seen at each time point and SEM images showed ECM deposition on the interior of the scaffold (Fig. 2). DNA content between low and high PCL were similar (Fig. 3A).

\section{Cell differentiation on low and high PCL}

ALP/DNA showed higher values for samples cultured in chondrogenic medium as opposed to osteogenic differentiation

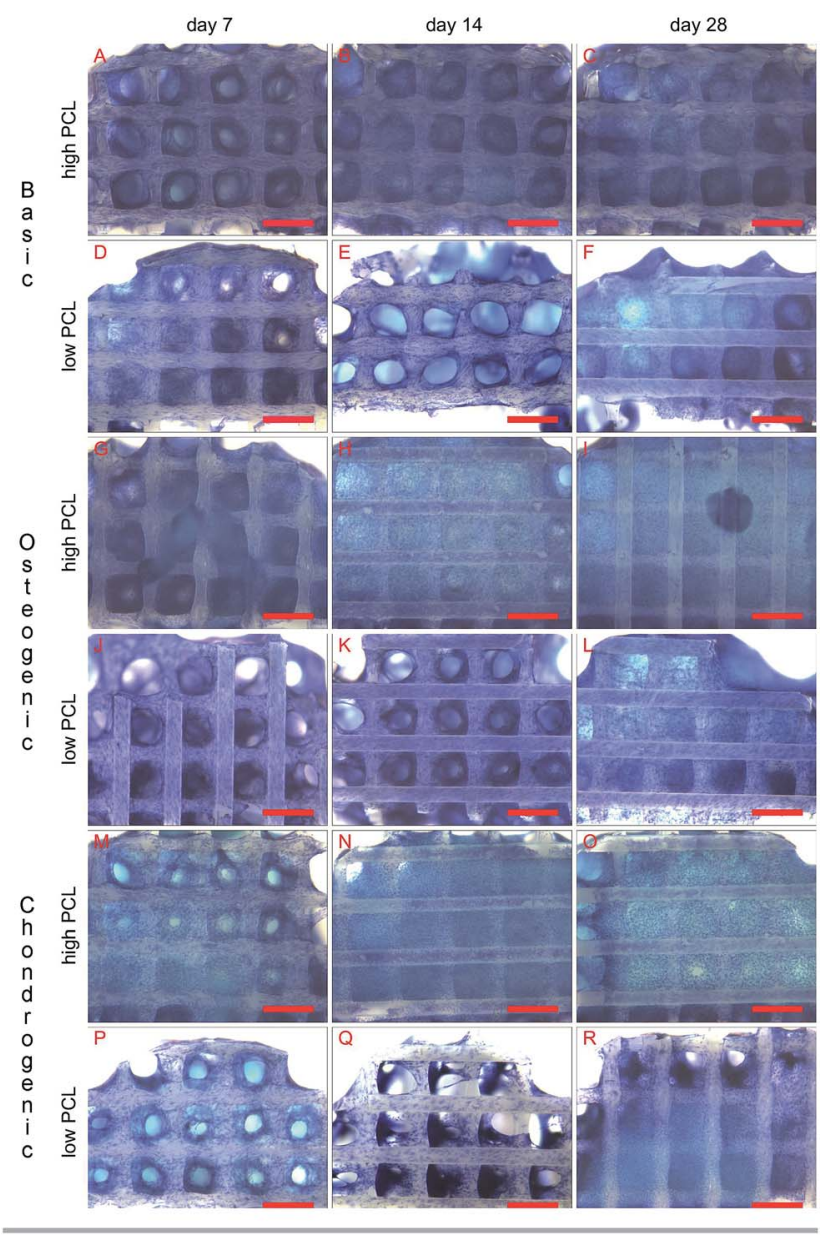

Fig. 1 Methylene blue staining of hMSC cultured on high PCL (A-C, $G-I$ and $M-O)$ and low PCL $(D-F, J-L$ and $P-R)$ in basic $(A-F)$, osteogenic $(G-L)$ and chondrogenic $(M-R)$ differentiation medium (scale bar is $1 \mathrm{~mm}$ ). 


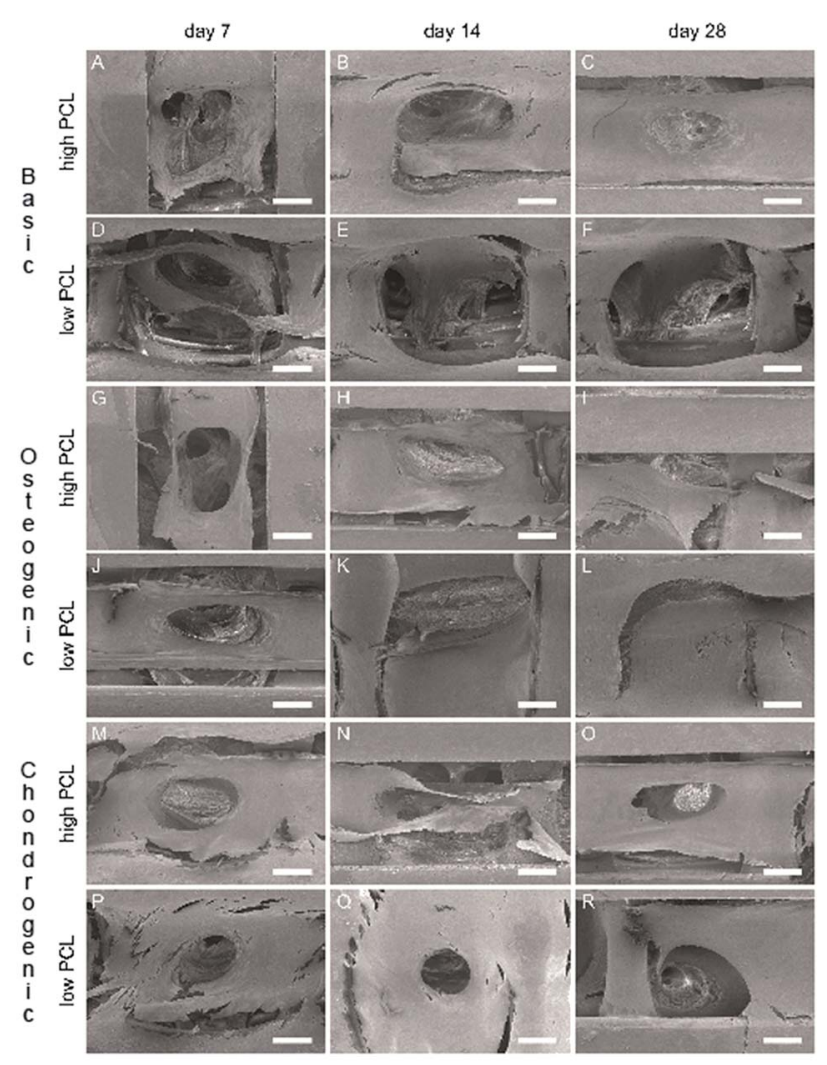

Fig. 2 SEM images hMSC cultured on high PCL (A-C, G-I and M-O) and low $P C L(D-F, J-L$ and $P-R)$ scaffolds in basic $(A-F)$, osteogenic $(G-L)$ and chondrogenic $(M-R)$ differentiation medium (scale bar is $200 \mu \mathrm{m})$

medium (Fig. 3B). It is only in chondrogenic media that the ALP/DNA values significantly increased in time for both PCL scaffolds. In high PCL scaffolds, after 28 days the ALP/DNA was statistically significant different between chondrogenic and osteogenic differentiation media. For low PCL scaffolds the results between chondrogenic and osteogenic differentiation media were statistically significant for day 14 and 28. The ALP/DNA results showed statistically significant higher values for low PCL scaffolds for chondrogenic differentiation media at day 14 and 28 compared to high PCL scaffolds. For cell seeded scaffolds cultured in osteogenic differentiation media, there was no statistically significant difference between high PCL and low PCL. The ALP staining corroborated the results of the biochemical analysis (Fig. 4). The trend seen in the biochemical analysis for basic media was reflected in the staining, with a peak at day 14. For cell seeded scaffolds cultured in osteogenic and chondrogenic differentiation media, the staining became more intense for day 14 and 28.

Chondrogenic differentiation was assessed by GAG production (Fig. 3C). GAG production was shown to increase statistically significant in time for cell seeded scaffolds cultured in chondrogenic differentiation media. Both low and high PCL supported chondrogenic differentiation as was also seen in the methylene blue images through the rounded cell morphology

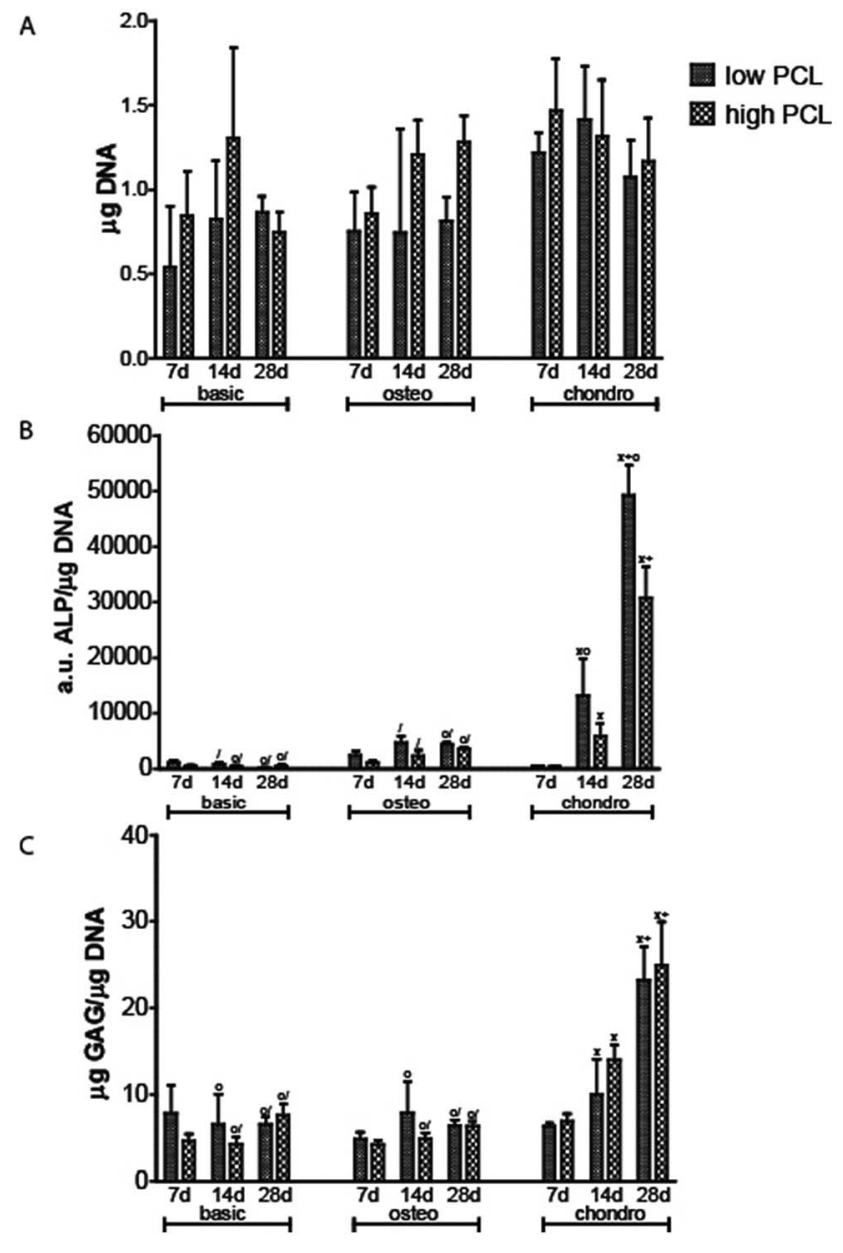

Fig. 3 DNA (A), ALP/DNA (B) and GAG/DNA (C) for hMSC cultured on low and high $P C L$ scaffolds cultured in basic, osteogenic and chondrogenic differentiation medium ( $X$ is statistical significant difference from day 7 in same media, + is statistical significant difference from day 14 in same media, $\mathrm{O}$ is statistical significant difference from high PCL chondro at same timepoint/statistical significant difference from low PCL chondro at same timepoint).

(Fig. 1M-R). GAG/DNA increased from $6.89 \pm 0.78 \mu \mathrm{g}$ GAG/ $\mu \mathrm{g}$ DNA at day 7 to $14.01 \pm 1.62 \mu \mathrm{g}$ GAG/ $\mu \mathrm{g}$ DNA at day 14 , to a maximum of $24.99 \pm 4.38 \mu \mathrm{g}$ GAG/ $\mu \mathrm{g}$ DNA at day 28 for high PCL scaffolds. Similar results were seen for low PCL scaffolds with $6.30 \pm 0.38 \mu \mathrm{g}$ GAG $/ \mu \mathrm{g}$ DNA at day $7,9.98 \pm 3.59 \mu \mathrm{g} \mathrm{GAG} / \mu \mathrm{g}$ DNA at day 14 , and a maximum of $23.55 \pm 5.24 \mu \mathrm{g}$ GAG/ $\mu \mathrm{g}$ DNA at day 28 as well. There was no statistical significant difference between low and high PCL scaffolds. The GAG/DNA values for scaffolds cultured in chondrogenic media were statistically significant higher than scaffolds cultured in basic and osteogenic media for day 14 and 28.

\section{Discussion}

Using a temporary or permanent structure, a scaffold, is one of the several strategies that exist to treat bone, chondral or osteochondral defects. The biomaterial for fabricating a 


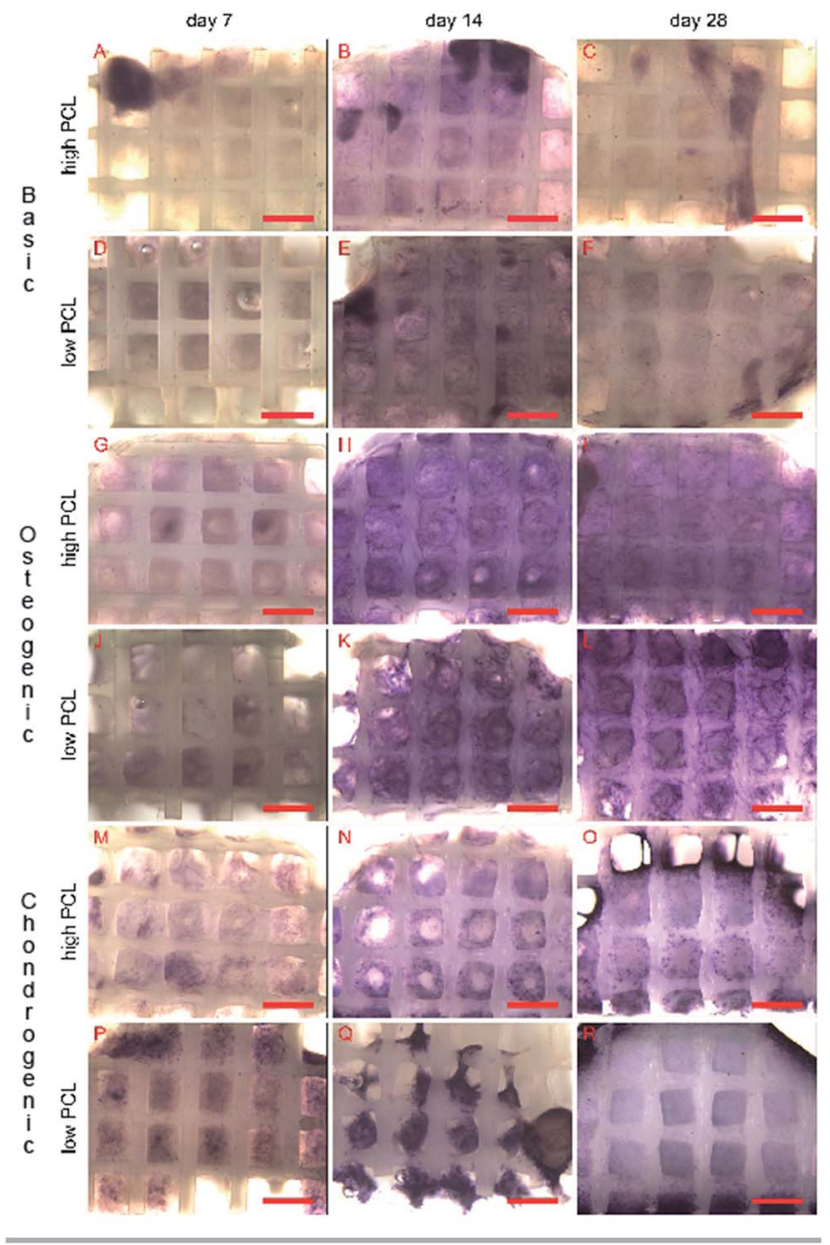

Fig. 4 ALP staining of hMSC cultured on high PCL (A-C, G-I and $M-O)$ and low $P C L(D-F, J-L$ and $P-R)$ scaffolds in basic $(A-F)$, osteogenic $(G-L)$ and chondrogenic $(M-R)$ differentiation medium (scale bar is $1 \mathrm{~mm}$ ).

scaffold can be either natural or synthetic. While ceramic and natural polymers can elicit a cellular response, synthetic polymers generally cannot do so. ${ }^{5,9,10}$ The tunable degradation time, degradation products and FDA approval for specific medical applications makes PCL an often used polymer. ${ }^{15,26-28}$ PCL is therefore available in a large number of different molecular weights. Engler and Discher among others, ${ }^{19,20}$ have shown with 2D hydrogels, coated with collagen, that cells were able to sense the stiffness of the hydrogel and determined their cell fate accordingly. Huebsch et al. showed this in $2 \mathrm{D},{ }^{21}$ but also in 3D hydrogels by encapsulating cells in alginate. ${ }^{22}$ By changing the molecular weight of PCL, the surface chemistry and topography remains the same but the bulk material stiffness will be different. Using AM scaffolds of PCL, the cells experience a different environment as they are not encapsulated and restricted in a certain morphology as they would in a hydrogel in absence of adhesive domains. We therefore investigated if the different molecular weight of PCL had an influence on cell behaviour in 3D scaffolds fabricated with similar structural properties, surface chemistry and topography but different bulk stiffness. Solid cylindrical samples were punched from compression moulded blocks and mechanically tested in an unconfined compression test. High PCL of 65.000 Da was stiffer than low PCL of 14.000 Da and thus showed a different bulk material stiffness with different molecular weights.

Methylene blue images of cell seeded scaffolds cultured in chondrogenic differentiation media, showed a rounded cell morphology suggesting the hMSCs differentiated towards the chondrogenic lineage (Fig. 1M-R). Biochemical analysis for chondrogenic differentiation showed a statistically significant increase in GAG/DNA in time for both low and high PCL (Fig. 3C). However, there was no statistical significant difference for the GAG/DNA levels between low and high PCL. The levels of GAG/DNA were higher than what reported in literature for in vitro cultured chondrogenically induced hMSCs. For an osteochondral scaffolds consisting of a PLA scaffold press coated with a chondrifying cell pellet, ${ }^{29}$ as well as for electrospun scaffolds seeded with hMSCs, ${ }^{30}$ a level around $5 \mu \mathrm{g}$ GAG/ $\mu \mathrm{g}$ DNA was reported.

ALP activity was performed as this is one of the many factors for the indication of hMSCs osteogenic differentiation (Fig. 3B). Previous work showed higher ALP/DNA values for cell seeded scaffolds cultured in chondrogenic differentiation medium compared to osteogenic differentiation medium. ${ }^{31}$ In the presented study, the ALP/DNA levels were again statistically higher for chondrogenic than osteogenic differentiation and basic medium. Interestingly, ALP/DNA was statistically significant higher for cell seeded scaffolds cultured in chondrogenic medium from low PCL compared to the osteogenic medium from low PCL and compared to the chondrogenic and osteogenic cultured cell seeded scaffolds from high PCL. ALP staining corroborated the biochemical analysis (Fig. 4). Together, these findings could implicate that the hMSCs induced to chondrogenic differentiation are going towards hypertrophy. From literature, it is known that hMSCs do have the tendency to differentiate towards a hypertrophic chondrogenic phenotype. $^{32,33}$ Jeong and Hollister ${ }^{34}$ had similar results with PCL scaffolds. However, they used chondrocytes and osteoblasts in their study. Although this effect has been seen in previous studies $^{32,33}$ and our own work (submitted), further analysis needs to be performed to further clarify if hMSCs induced to the chondrogenic lineage are indeed going towards an hypertrophic phenotype.

Additionally, the effect of bulk material stiffness on cell behaviour in a 3D open porous structure could be investigated. With AM the scaffold architecture was completely controlled. Roosa et al. ${ }^{35}$ showed with PCL scaffolds that a pore size in the range of $350-800 \mu \mathrm{m}$ resulted in differences in bone regeneration and mechanical properties after 4 weeks of subcutaneous implantation in mice. However, no beneficial effects were detectable anymore after implantation for 8 weeks, even though the porosity of the PCL scaffolds used were not the same. Hsu et al. ${ }^{11}$ created scaffolds with a pore size of $250 \mu \mathrm{m}$ through fused deposition modelling. They changed the angle between successive layers, effectively changing the pore shape. Additionally, they made square scaffolds and scaffolds which were concentric with a large free inner space. The layer angle did not 
result in different cellular responses, but the concentric scaffolds showed more collagen deposition by the seeded osteoblasts.

By using AM we could additionally investigate if the bulk material stiffness had an influence in 3D open porous scaffolds. It is known that encapsulated cells in hydrogels do react to stiffness. Yet, no study has investigated if this is also the case for open porous 3D scaffolds. Changing the bulk stiffness could be easily performed by using a different polymer. This will, however, result in a different surface chemistry and topography as well, which are biomaterial characteristics known to have the ability to influence cell behaviour. ${ }^{36,37}$ Using the same polymer, PCL, but different molecular weights, the bulk stiffness was changed maintaining surface chemistry and topography unaltered.

Most reported studies that investigate the influence of biomaterial stiffness on cell differentiation were conducted with hydrogels. ${ }^{19-23}$ The stiffness range for instructing differentiation was reported to be in the range of 1-100 kPa for 2D hydrogels coated with collagen and 3D hydrogels where cells were encapsulated. In our situation the stiffness of the bulk material far exceeds the stiffness of the hydrogels. Another fundamental difference is that cells were not encapsulated but attached to the scaffold surface. The deposited ECM accumulates over time creating a distance from the surface of the scaffold. It is known that cells can sense up to a certain distance the stiffness, which would be at some point in time the stiffness of the deposited ECM. ${ }^{38,39}$ As demonstrated by Chaudhuri et $a .^{21}$ not only the stiffness of the material is important but also the ligand density, the cell-ECM anchoring. They showed that the available ligand density and the opportunity for cells to actively remodel their environment were also able to direct cell differentiation. In our 3D AM scaffolds, the ECM secreted by the cells can be remodelled without restrictions imposed by the scaffold system, in contrast to cells encapsulated in hydrogels.

Scaffolds were coated with collagen type I before cell seeding. This means that cells were not directly interacting with the PCL surface, which may influence the actual surface stiffness that is perceived by the cells. However, Trappmann et $a l^{40}$ reported that the stiffness as sensed by cells mainly depends on the protein-biomaterial surface interactions. With more binding sites between the protein and the surface, less deformation of the protein is possible and cells sense a higher stiffness of the biomaterial surface. Less binding sites between surface and proteins result in cells perceiving a lower stiffness. In this study, by using the same polymer with different molecular weights, the same surface chemistry and therefore the same surface-protein interaction can be expected. As such, the influence of different surface-protein interaction should not play a significant role, and differences in the stiffness perceived by the cells will be based on differences in stiffness of the biomaterial.

The cells seeded on low PCL had a statistically significant higher ALP/DNA level than high PCL. Currently, the mechanism that causes the observed difference is not known. More research should be conducted to unravel the cause of the underlying mechanism responsible for the higher ALP/DNA levels. To assess the chondrogenic and osteogenic differentiation of the hMSCs, the common markers GAG and ALP were measured. In future experiments, PCR and immunohistological staining should be performed to further discriminate between the differentiation lineages and between the low and high PCL scaffolds. For osteogenic differentiation bone sialoprotein, BMP2, osteocalcin, osteopontin and osteonectin would be good markers, while for chondrogenic differentiation collagen typeII, collagen type-X, SOX-9, and aggrecan would be logical choices. Collagen type-X could give indication if the chondrogenic differentiation is indeed going towards hypertrophy as expected by the high GAG/DNA coupled with the high ALP/DNA ratio. Performing long term cultures (e.g. 8 weeks) should indicate if the current observations will be sustained on a longer time period. It would be interesting to investigate if a molecular weight of PCL higher than 65.000 Da would result in lower ALP/DNA values and what would be the cause of this phenomenon. The difference in bulk mechanical stiffness by using a different molecular weight could be very useful in designing scaffolds. The relation between pore size, shape and porosity on the apparent stiffness of the scaffold is described in literature. ${ }^{41-43}$ Pore structural properties could be, therefore, an extra variable available to customize the scaffold design to meet the desired criteria, for example matching scaffold mechanical properties to the native tissue mechanical properties. The effect of bulk stiffness and scaffold architecture could be used in a mechanically loading bioreactor, for example, to optimize and precondition tissue engineering scaffolds. The design of the scaffold could be further optimized by coupling such mechanical loading with numerical modelling able to predict cell differentiation. ${ }^{24}$

\section{Conclusions}

Two extremes of molecular weights for PCL were chosen, 14.000 Da and 65.000 Da. Mechanical analysis showed a higher stiffness for the high molecular weight PCL. Cell seeded scaffolds of low and high PCL were cultured in basic, osteogenic and chondrogenic differentiation medium. In both low and high PCL the GAG/DNA levels were high, showing a robust chondrogenic differentiation. Interestingly, low PCL had higher levels of ALP/DNA than high PCL suggesting a stronger tendency for low PCL to go into hypertrophy. Further research should aim at unravelling the cause for this phenomenon as the results could be used to design site specific regions in an osteochondral scaffold.

\section{Acknowledgements}

We would like to acknowledge funding for W.H. from the Netherlands Institute of Regenerative Medicine, contract grant number: FES0908. The research of J.R. was financially supported by a VENI grant by the Dutch Technology Foundation STW. We also gratefully acknowledge Andrea Sanfelice for his preparatory work. 


\section{References}

1 J. F. Mano and R. L. Reis, J. Tissue Eng. Regener. Med., 2007, 1, 261-273.

2 M. T. Arafat, C. X. F. Lam, A. K. Ekaputra, S. Y. Wong, X. Li and I. Gibson, Acta Biomater., 2011, 7, 809-820.

3 F. W. Janssen, J. Oostra, A. V. Oorschot and C. A. van Blitterswijk, Biomaterials, 2006, 27, 315-323.

4 H. Yuan, H. Fernandes, P. Habibovic, J. de Boer, A. M. C. Barradas, A. de Ruiter, W. R. Walsh, C. A. van Blitterswijk and J. D. de Bruijn, Proc. Natl. Acad. Sci. U. S. A., 2010, 107, 13614-13619.

5 P. V. Giannoudis, H. Dinopoulos and E. Tsiridis, Injury, 2005, 36, S20-S27.

6 N. Zhang and D. H. Kohn, Birth Defects Res., Part C, 2012, 96, 63-81.

7 G. D. Zhou, W. Liu, L. Cui, X. Y. Wang, T. Y. Liu and Y. L. Cao, Tissue Eng., 2006, 12, 3209-3221.

8 D. W. Hutmacher, J. Biomater. Sci., Polym. Ed., 2001, 12, 107124.

9 T. Cordonnier, J. Sohier, P. Rosset and P. Layrolle, Adv. Eng. Mater., 2011, 13, B135-B150.

10 A. J. Salgado, O. P. Coutinho and R. L. Reis, Macromol. Biosci., 2004, 4, 743-765.

11 S.-H. Hsu, H.-J. Yen, C.-S. Tseng, C.-S. Cheng and C.-L. Tsai, J. Biomed. Mater. Res., Part B, 2007, 80, 519-527.

12 J. S. Lee, H. D. Cha, J. H. Shim, J. W. Jung, J. Y. Kim and D. W. Cho, J. Biomed. Mater. Res., Part A, 2012, 100, 18461853.

13 L. Shor, S. Güçeri, R. Chang, J. Gordon, Q. Kang, L. Hartsock, Y. An and W. Sun, Biofabrication, 2009, 1, 015003.

14 I. Zein, D. W. Hutmacher, K. C. Tan and S. H. Teoh, Biomaterials, 2002, 23, 1169-1185.

15 M. A. Woodruff and D. W. Hutmacher, Prog. Polym. Sci., 2010, 35, 1217-1256.

16 J. Malda, T. B. F. Woodfield, F. van der Vloodt, C. Wilson, D. E. Martens, J. Tramper, C. A. van Blitterswijk and J. Riesle, Biomaterials, 2005, 26, 63-72.

17 T. B. F. Woodfield, J. Malda, J. de Wijn, F. Péters, J. Riesle and C. A. van Blitterswijk, Biomaterials, 2004, 25, 4149-4161.

18 A. Buxboim, I. L. Ivanovska and D. E. Discher, J. Cell Sci., 2010, 123, 297-308.

19 D. E. Discher, Science, 2005, 310, 1139-1143.

20 A. Engler, S. Sen, H. Sweeney and D. Discher, Cell, 2006, 126, 677-689.

21 O. Chaudhuri, L. Gu, M. Darnell, D. Klumpers, S. A. Bencherif, J. C. Weaver, N. Huebsch and D. J. Mooney, Nat. Commun., 2015, 6, 6364.

22 N. Huebsch, P. R. Arany, A. S. Mao, D. Shvartsman, O. A. Ali, S. A. Bencherif, J. Rivera-Feliciano and D. J. Mooney, Nat. Mater., 2010, 9, 518-526.

23 N. S. Hwang, S. Varghese, H. Li and J. Elisseeff, Cell Tissue Res., 2011, 344, 499-509.
24 W. J. Hendrikson, C. A. van Blitterswijk, N. Verdonschot, L. Moroni and J. Rouwkema, Biotechnol. Bioeng., 2014, 111, 1864-1875.

25 J. D. de Bruijn, I. van den Brink, S. Mendes, R. Dekker, Y. P. Bovell and C. A. van Blitterswijk, Adv. Dent. Res., 1999, 13, 74-81.

26 R. M. Baker, J. H. Henderson and P. T. Mather, J. Mater. Chem. B, 2013, 1, 4916-4920.

27 J. S. Park, D. G. Woo, B. K. Sun, H. M. Chung, S. J. Im, Y. M. Choi, K. Park, K. M. Huh and K. H. Park, J. Controlled Release, 2007, 124, 51-59.

28 S. Park, S. Lee and W. Kim, Bioprocess Biosyst. Eng., 2011, 34, 505-513.

29 R. Tuli, S. Nandi, W. J. Li, S. Tuli, X. X. Huang, P. A. Manner, P. Laquerriere, U. Noth, D. J. Hall and R. S. Tuan, Tissue Eng., 2004, 10, 1169-1179.

30 J. K. Wise, A. L. Yarin, C. M. Megaridis and M. Cho, Tissue Eng., Part A, 2009, 15, 913-921.

31 W. J. Hendrikson, X. Zeng, J. Rouwkema, C. A. van Blitterswijk, E. van der Heide and L. Moroni, in revision.

32 M. B. Mueller, M. Fischer, J. Zellner, A. Berner, T. Dienstknecht, L. Prantl, R. Kujat, M. Nerlich, R. S. Tuan and P. Angele, Cells Tissues Organs, 2010, 192, 158-166.

33 C. K. Abrahamsson, F. Yang, H. Park, J. M. Brunger, P. K. Valonen, R. Langer, J. F. Welter, A. I. Caplan, F. Guilak and L. E. Freed, Tissue Eng., Part A, 2010, 16, 3709-3718.

34 C. G. Jeong and S. J. Hollister, Biomaterials, 2010, 31, 43044312.

35 S. M. M. Roosa, J. M. Kemppainen, E. N. Moffitt, P. H. Krebsbach and S. J. Hollister, J. Biomed. Mater. Res., Part A, 2010, 92, 359-368.

36 Y. Mei, K. Saha, S. R. Bogatyrev, J. Yang, A. L. Hook, Z. I. Kalcioglu, S. W. Cho, M. Mitalipova, N. Pyzocha, F. Rojas, K. J. van Vliet, M. C. Davies, M. R. Alexander, R. Langer, R. Jaenisch and D. G. Anderson, Nat. Mater., 2010, 9, 768-778.

37 J. Zhang, X. Luo, D. Barbieri, A. M. C. Barradas, J. D. de Bruijn, C. A. van Blitterswijk and H. Yuan, Acta Biomater., 2014, 10, 3254-3263.

38 M. J. Dalby, N. Gadegaard, R. Tare, A. Andar, M. O. Riehle, P. Herzyk, C. D. W. Wilkinson and R. O. C. Oreffo, Nat. Mater., 2007, 6, 997-1003.

39 S. Sen, A. J. Engler and D. E. Discher, Cell. Mol. Bioeng., 2009, 2, 39-48.

40 B. Trappmann, J. E. Gautrot, J. T. Connelly, D. G. T. Strange, Y. Li, M. L. Oyen, M. A. Cohen Stuart, H. Boehm, B. Li, V. Vogel, J. P. Spatz, F. M. Watt and W. T. S. Huck, Nat. Mater., 2012, 11, 642-649.

41 L. Moroni, J. R. de Wijn and C. A. van Blitterswijk, J. Biomed. Mater. Res., 2005, 75, 957-965.

42 L. Moroni, J. R. de Wijn and C. A. van Blitterswijk, Biomaterials, 2006, 27, 974-985.

43 L. Moroni, G. Poort, F. van Keulen, J. R. de Wijn and C. A. van Blitterswijk, J. Biomed. Mater. Res., 2006, 78, 605-614. 